\title{
Hypothetical Dark Matter/Axion Rockets: And the Neutrinos without SUSY Problem
}

\author{
Andrew Walcott Beckwith \\ Physics Department, College of Physics, Chongqing University Huxi Campus, Chongqing, China \\ Email: Rwill9955b@gmail.com, abeckwith@uh.edu
}

How to cite this paper: Beckwith, A.W. (2016) Hypothetical Dark Matter/Axion Rockets: And the Neutrinos without SUSY Problem. Journal of High Energy Physics, Gravitation and Cosmology, 2, 457-466.

http://dx.doi.org/10.4236/jhepgc.2016.24039

Received: July 19, 2016

Accepted: August 20, 2016

Published: August 23, 2016

Copyright $\odot 2016$ by author and Scientific Research Publishing Inc. This work is licensed under the Creative Commons Attribution International License (CC BY 4.0).

http://creativecommons.org/licenses/by/4.0/

\section{(c) (i) Open Access}

\begin{abstract}
We present Dark Matter candidates from non SUSY processes, in a way emphasizing how a Dark Matter (DM) candidate of roughly $100-400 \mathrm{GeV}$ could be formed. As has been said about the Photon rocket and Axions rockets, the presence of a magnetic field supposedly would switch DM particle candidates to photons, in such a way as to in the end configure a photon rocket style device from DM in a thrust chamber. The presence of Dark Matter (DM) would in itself merely indicate that the emerging photon thrust would be comparatively greater than it would be for more conventional photon rockets. This amplifies and improves upon a so called axion rocket ram jet for interstellar travel. We assume that much the same sort of methodology for a would-be axion ramjet could be employed for DM, with perhaps greater thrust/power conversion efficiencies.
\end{abstract}

\section{Keywords}

Dark Matter, Photon Rocket, Axions

\section{Introduction}

We will attempt to discuss the presence of Dark Matter (DM) and candidates for Axions from non SUSY processes, in ways we think would tie into their discovery via the (Linear Hadron Collider) LHC. Once vetted and confirmed by LHC measurements, we can eventually talk about the feasibility of a DM/Axion ramjet. We reference material brought up by the author in IDM 2008 [1], material discussed by Hooper [2] which was initially in (Identification of Dark Matter) IDM 2008, but which is an arxiv entry now [3], Meissner and Nicholai neutrino physics, without SUSY [4] article, and if one assumes commonality of DM with variants of Neutrino physics [5], we can also discuss a solution to the conundrums brought up by Wired Magazine [6] as to a DM 
ram jet, which is further elaborated upon in [7] [8]. We conclude with a discussion as to the fact that Corda in [9] as well as S. Nojiri, S. D. Odintsov [10], brought up alternatives, as well as in Dark Matter which are also an alternative to the possible formation of (sterile) neutrinos, without the use of SUSY [4] article, which the author brings up, since the ram jet model as brought up is assuming hinges upon dark matter, as far as a propulsion protocol, to get about the problems brought up in [6].

\section{What Can Be Said about Axions?}

The author estimates that at near-light speeds, the available axion-power would be about 3 watts $/ \mathrm{cm}^{2}$ times $\beta \times \gamma^{2}$ where $\beta=(v / c)$ is the velocity relative to light, and $\gamma^{2}=1 /\left[1-\beta^{2}\right]$ [7] [8] is the square of the relativistic mass-increase factor. At a velocity of $99.9 \% \mathrm{c}$, the available power from axions would be about $1500 \mathrm{watts} / \mathrm{cm}^{2}$, enough power for a modest energy-efficient space drive. And the faster we go, the more such power becomes available. Note though that this is a long way from saying that we are having a viable interstellar vehicle candidate. We are saying that in principle that a Photon rocket MAY be improved upon, and that this DM/Axion [11] destruction via intense $\mathrm{E} \& \mathrm{~B}$ fields is an avenue toward making a more powerful Photon rocket. We are leaving as a full blown $\mathrm{R}$ and $\mathrm{D}$ project the feasibility of obtaining Axions/DM as part of phase transitions [12] [13] in the first place, which will be the last part of our article.

And, Axions [14] are one of the components of the DM candidates we will bring up. To do this, we will review the contents the author presented in IDM 2008, with a goal toward isolating neutrino/Axion/DM candidates [14], as well as the available masses of DM, and of Neutrino candidates. The key point we will raise is this: we wish to improve what is known as a Photon rocket, i.e., propulsion without conventional propulsion. We will speak of the different DM and axion candidates, select the candidate we think is most pertinent to propulsion, and then discuss the basic physics of the photon rocket, with an explanation of how to upgrade the photon rocket. The axion rocket concept is the modus operandi we will follow. i.e., a large magnetic field in a chamber can change axions to giving photonic components. Due to constraints upon mass we can carry, we favor the concept similar to axion ram jet for interstellar travel. i.e., we wish to find a ram jet that has greater thrust than the photon rocket, and better than the axion rocket itself. Using propulsion candidates similar to DM may provide a way to gain further improvements to the axion rocket, which in itself as a ram jet is an improvement over both the simple photonic rocket and the axion ram jet rocket.

\section{Reviewing the Highlights of the IDM 2008 Talk: Abandoned DM Candidates, Leading to Present Candidates for DM?}

We will in this section give reasons as to why the particular candidate for DM which we will invoke for the paper was used. Doing so requires that we discuss why some of the baryonic and non baryonic candidates for DM have been recently largely abandoned. 
We will briefly define some of the known candidates for dark matter which have been abandoned. The first of them is the MACHO [15]. Briefly put, MACHOs are DM candidates with masses up to one tenth of a solar mass. i.e., the Macho concept heavily relies upon relatively inert matter galactic baryonic dark matter seem most likely to be in the form of compact objects and could be in one of two mass windows: either in the brown dwarf regime or in the mass range corresponding to supermassive black holes. Muramaya [15] (2006) asserts as do others at the IDM 2008 conference that what is known as a gravitational lens phenomena eliminates them as a viable DM candidate. We should remember in thinking about MACHOs we are referring to a baryonic candidate for DM. i.e., relatively normal matter. Gravitational lensing [16] occurs when a brown dwarf or a black hole passes between a light source, such as a star or a galaxy, and an observer on the Earth. The object focuses the light rays, causing the light source to brighten [17] Astronomers diligently search photographs of the night sky for the telltale brightening that indicates the presence of a MACHO. And this is for reasons which were brought up in IDM 2008, and which Muramaya (2006) indicates were why MACHO's were finally eliminated as a serious candidate [15].

Next, we can look at what is known as standard/sterile neutrinos as a DM candidate [18]. The idea being that the sterile neutrino with the mass in the keV range might answer some DM parameter problems which have dogged astrophysicists. Adding 3 righthanded (sterile) neutrinos to the Standard Model (SM) can solve several "beyond the Standard Model" problems within one consistent framework. Great, except that they are pretty much ruled out via the fact that its mass is so small, and that the TremaineGunn argument [19] to the effect that "For Neutrinos to dominate the halo of dwarf galaxies, one would need to pack them so much that the Pauli Exclusion principle would be violated." Traditional neutrino masses, if Warm Dark Matter [20] existed would interfere with structure formation in the early universe. i.e., we can state that if we look at neutrinos moving at nearly the speed of light, that would interfere with structure formation, often erase it at small scales. This notwithstanding what the author views as the mistake made by Ruchayskiy's overly optimistic view of sterile neutrino as a DM candidate [21].

So what about Champs? M. Taoso, G, Bertone and A. Masiero (2008) [22] give a list of requirements for non baryonic DM, a ten point test litany for a non baryonic DM candidate to match up to. We will reproduce it here. "Namely: An extraordinarily rich zoo of non-baryonic dark matter candidates has been proposed over the last three decades. Here we present a ten-point test that a new particle has to pass in order to be considered a viable DM candidate. (I) Does it match the appropriate relic density? (II) Is it cold? (III) Is it neutral? (IV) Is it consistent with BBN? (V) Does it leave stellar evolution unchanged? (VI) Is it compatible with constraints on self-interactions? (VII) Is it consistent with direct DM searches? (VIII) Is it compatible with gamma-ray constraints? (IX) Is it compatible with other astrophysical bounds? (X) Can it be probed experimentally?"

Champs, specifically massive, charged DM candidates, are largely ruled out due to 
their excessive mass, and also due to energy levels between 1 to $1000 \mathrm{TeV}$. We urge the readers to read M. Taoso, G, Bertone and Masiero's (2008) paper [22]. The main objection is that CHAMPS have many similarities to heavy hydrogen, and that we would see traces of them in the ocean. Suffice to say no such traces have been detected. Assume to the contrary, that CHAMPS may still be a viable candidate, we would be up against the datum that the proposed particles would weigh at least 100,000 times the mass of the proton, too heavy to be created by the world's most powerful particle accelerator, the Large Hadron Collider. Furthermore, CHAMPS would be too massive to produce experimentally detectable light, or electro magnetic radiation, even in a magnetic field in space.

Still, if CHAMPs are negatively charged, they might have bound to iron and other elements to create supermassive varieties that could be detected by their weight. These elements might also absorb and emit telltale X-rays that could be observed by telescopes

This leads to a long shot for CHAMPS. Not completely ruled out, but still very difficult to observe. So we have chosen other candidates to consider.

So we deal with cold dark matter as our DM/Axion candidate basis for starting this inquiry. To do so, we reference what the author presented in IDM 2008 about DM, a takeoff of Meissner and Nicholai's (2008) [4] work on non-standard neutrino physics. As the author reported in IDM 2008, this leads to a DM candidate which we report having a mass value of between 300 to over $400 \mathrm{GeV}$, which fits in strictures that $\mathrm{Mu}$ ramaya (2006) [15] gave in his Les Houches lecture on extensions of the standard model and DM physics.

\section{Meissner \& Nicolai: Extending the Standard Model}

With classically conformal Langrangian, with the usual Higgs doublet and one extra weak scalar field: This leads to a statement about the existence of the so called Majoran candidate for an axion candidate, without invoking SUSY [4]

$$
\tilde{\varphi}(x)=\varphi(x) \cdot \exp \left[\frac{i a(x)}{\sqrt{2} \mu}\right]
$$

The expression $a(x)$ yields a pseudo-Goldstone particle associated with "spontaneous breaking of a new global (modified Lepton number) symmetry". And this $a(x)$ shares properties with the axion. This is partly due to conformal symmetry eliminating the existence of conformal Lagrangian contributions. So we get masses for particles like neutrinos-heavier than the SUSY neutrino candidate, but having the same "branching ratio" $(a(x)$ is massless). In our treatment of this problem, we assume that Meisssner and Nicholai [4] are almost right. i.e., that the axion is $10^{-9}$ the rest mass of an electron in $\mathrm{GeV}$ value. But that what they calculated is close enough to be still with value and merit to review.

Meissner and Nicholai (2008) [4] worked with a classically conformal Lagrangian model for which [4] 


$$
\varsigma_{\text {int }}=S M-\frac{\lambda_{1}}{4} \cdot\left(\Phi^{+} \Phi\right)^{2}-\frac{\lambda_{2}}{2}\left(\Phi^{+} \Phi\right)\left(\phi^{+} \phi\right)+\frac{\lambda_{3}}{4}\left(\phi^{+} \phi\right)^{2}
$$

We begin first with an interaction Lagrangian with the usual Higg's doublet $\Phi$ and an extra weak singlet complex scalar field $\phi(x)$. We minimize the effective potential varying values of the effective coupling constants to

$$
\left\{\lambda_{1}, \lambda_{2}, \lambda_{3}\right\} \underset{\text { effective-coupling-const-values }}{\longrightarrow}\{1,447,0.648,0.871\} .
$$

We also consider non-zero expectation values, if we remove the $\mathrm{O}(6)$ symmetry, which would lead to values of

$$
\left\{\lambda_{1}, \lambda_{2}, \lambda_{3}, g_{t}, Y_{M}^{2}\right\} \underset{\text { vanishing-non-zero-vacuum-expectation-values }}{\longrightarrow}\{3.77,0.3 .72,3.73,1,0.4\} .
$$

Then we work with $H=\sqrt{\Phi^{+} \Phi}$, and deal with the case when the $\phi$ carries lepton charge. Then if we assume h.c. is the Hermitian conjugate, we make the following identifications in the Lagrangian: $Q^{i}$ and $L^{i}$ are the left-chiral quark and lepton doublets, $U^{i}$ and $D^{i}$ are the right-chiral up- and down-like quarks; while $E^{i}$ are right-chiral electron-like leptons, and $N^{i}=v_{R}^{i}$ are right-chiral neutrinos. Looking at real versions of the Yukawa matrices $Y_{i j}^{E}, Y_{i j}^{U}$, and $Y_{i j}^{M}$, and doing some CKM matrix style decompositon, we find that the Lagrangian represented in Equation (1) and with the SM terms as given by Equaution (2) will admit two global U(1) symmetries, so that both the standard baryon symmetry and modified lepton number symmetry $U(1)_{L}$ to set the template for numerical minimization, which lead to making the parameterized values as

$$
\left\{\lambda_{1}, \lambda_{2}, \lambda_{3}, g_{t}, Y_{M}^{2}\right\} \longrightarrow s\{3.77,0.3 .72,3.73,1,0.4\}
$$

This is a mixture of symmetry arguments and numerical minimization of the parameter space, using [4]:

$$
\begin{aligned}
S M & =\left(\overline{L^{i}} \Phi Y_{i j}^{E} E^{j}+\bar{Q}^{i} \Phi Y_{i j}^{D} D^{j}+\bar{Q}^{i} \varepsilon \cdot \Phi^{*} Y_{i j}^{U} U^{j}\right. \\
& \left.+\overline{L^{i}} \varepsilon \cdot \Phi^{*} Y_{i j}^{v} N^{j}+\phi \cdot N^{i T} C^{-1} Y_{i j}^{M} N^{j}+\text { h.c. }\right)
\end{aligned}
$$

This will lead to conformal symmetry reduction of the Classical conformal Lagrangian due to power zero to power 2 terms do not arise in the conformal Lagrangian, while we would say they are normally expected in this type of Lagranginan. In addition the Higgs boson would not be needed since it would break the conformal symmetry of Equation (2) above, i.e., of this Lagrangian. Equation (3) is important in the resultant current calculations and was an aid to us getting the DM bound as given in Equation (5) below.

\section{Parameter Space Treatment in Order to Isolate a DM Candidate}

Meissner and Nicholai [4] eventually obtained the following averaged out parameter space values, namely 


$$
\begin{aligned}
& \lambda_{1}=3.77, \lambda_{2}=3.72, \lambda_{3}=3.73 \\
& \langle H\rangle=\left\langle\sqrt{\Phi^{+} \Phi}\right\rangle=174 \mathrm{GeV},\langle\varphi\rangle=958 \mathrm{GeV} \\
& H^{\prime}=H \cos \beta+\varphi \cdot \sin \beta \\
& \varphi^{\prime}=-H \cdot \sin \beta+\varphi \cos \beta \\
& m_{H^{\prime}}=207 \mathrm{GeV}, m_{\varphi^{\prime}}=477 \mathrm{GeV}, \sin \beta=0.179
\end{aligned}
$$

The value of $m_{\varphi^{\prime}}=477 \mathrm{GeV}$ so obtained is the very upper limit of DM candidate which was brought up by many of IDM 2008's DM speakers. Eventually, leading to what the author numerically estimated a candidate DM candidate mass to be of the order of

$$
m_{\varphi^{\prime}}>\vartheta(\text { order }) 400 \mathrm{GeV}
$$

This is an upper value of the specified DM mass. Researchers in FNAL theoretical astro physics in IDM 2008 namely Dan Hooper [3] specified an indirect detection scheme for DM leading to a mass range for WIMP candidates below $200 \mathrm{GeV}$, with a DM WIMP mass being greater than $200 \mathrm{GeV}$ leading to a quark and gluon de composition and decay of the WIMPs. So their scheme as presented in IDM 2008 favored masses within an order of magnitude of $100 \mathrm{GeV}$. So if we eliminate ultra massive DM candidates this way, and get masses within the range, say of 100 to 400 or so GeV, with favored values likely within sight of $100 \mathrm{GeV}$, we can now talk about what the behavior of an Axion (Majoran) DM ramjet would be, and why it is likely obeying about 1500 watts $/ \mathrm{cm}^{2}$, enough power for a modest energy-efficient space drive.

\section{First Principles of an Axion/DM Ramjet}

We should state specifically that we are thinking of converting axion/DM "particles" to, after intersecting them with a magnetic field into photons. i.e., we are improving upon the specifications for a Photon rocket drive. Let us first review a few basics of the photon rocket, then go to how to convert axions/DM to photons.

Currently proposed photon rocket designs include the Nuclear Photonic Rocket and the Antimatter Photonic Rocket (first proposed by Eugen Sanger in the 1950s) [23]. In a Nuclear Photonic Rocket, a nuclear fission reactor is used to directly heat tungsten coils or graphite blocks to white-heat at the focus of a parabolic reflector. While using a laser to produce the light beam would provide much better collimation, this is offset by the reduction in efficiency incurred by powering a laser rather than using black-body radiation directly (a nuclear fission reactor will generally output at least 5 to 10 times more energy as heat than it can the electricity it could generate).

Now, we can talk about a photon rocket in terms of destruction of DM/Axions via intense $\mathrm{E} \& \mathrm{M}$ fields. Note that in doing this we are paying attention to the Wired (2008) [6] article purporting to show that as quoted from the article: "And then there's the issue of fuel. It would take at least the current energy output of the entire world to send a probe to the nearest star, according to Brice N. Cassenti [6], an associate professor with the Department of Engineering and Science at Rensselaer Polytechnic Insti- 
tute. That's a generous figure: More likely, Cassenti says, it would be as much as 100 times that". So, we can only talk about perhaps a ram jet engineering construction, i.e., scooping up Axions/DM from the interstellar void and using that as a fuel source. So how do we get around this? It so happens that the mass values as ascertained above in the authors IDM 2008 meeting presentation, of perhaps up to several hundred $\mathrm{GeV}$ is the only way possible to get high frequency.

As can be inferred from P. Sikivie (1983) [24], "Every axion which is converted to a photon with the same total energy and going in the same direction produces a momentum kick of

$$
\Delta p=m c \times \gamma \cdot(1-\beta)
$$

where $m$ is the axion rest mass." If we make a swap between axions and DM, or use a mass of several hundred $\mathrm{GeV}$ as a starting point due to the calculations so referenced above and avoid the absurdity mentioned in the Wired (2008) article, i.e., not think of carrying feul to the stars, but use the ram jet modus operandi, we can possibly think of obtaining a working, upgraded Photon rocket which would improve our chances of greatly improved space propulsion.

For a DM rocket, if the DM has some of the axion properties, this would likely mean working with velocities nearer to. $0.1 \%$ (light-speed), not the near $c$ values spoken above. The main point of first of all confirming a viable candidate for the DM particle, after we try to confirm some of the DM physics would be in getting a realistic propulsion candidate so we could get perhaps a ship of the order of magnitude of at least an air craft carrier in bulk to travel at one tenth the speed of light. Furthermore, before we do this, it would be helpful if we confirmed a prediction given by Meissner and Nicholai (2008), namely, the coupling of the Meissner Axion candidate to photons, of the order of [4]

$$
f_{a}=\vartheta\left(10^{15} \mathrm{GeV}\right)
$$

Either this energy release, if handled appropriately, and/or a DM candidate merely improving the so called axion ram jet energy release figure given in the estimates above would begin to yield a more practical candidate for improving the efficiency of a photon rocket and/or a ramjet based on axion conversion, via strong magnetic fields into a powerful light source beam.

\section{Conclusions}

Our short article has focused upon several themes. First, in IDM 2008, the author predicted a mass range DM up to about $400 \mathrm{GeV}$, per particle, and received vetting of this prediction from Dan Hooper [3] who specified a preferred $100-200 \mathrm{GeV}$ range for DM candidates, for reasons stated in the manuscript. If Axions are indeed roughly equivalent to the DM candidates, this mass range in itself adds credibility toward implementation of Equation (4), leading credence to the authors estimation of a thrust value of for DM production if we approach $V=C$ (the speed of light) of $10^{14} \times 3$ watts $/ \mathrm{cm}^{2}$ times $\beta \times \gamma^{2}$. 
We avoid the absurdity of the idea of carrying an energy supply of the magnitude of the Earths entire energy output with the space craft for a journey to the stars. However, the real engineering problems lie ahead in a radical upgrade of the Proton rocket ship. i.e., photon rockets provide the maximum exhaust velocity (c) and the minimum exhaust mass (zero); they represent the theoretical maximum in specific impulse, but provide very low thrust, given by $2 \mathrm{PR} / \mathrm{c}$, where $\mathrm{P}$ is the emission power in watts and $\mathrm{R}$ is the efficiency of the reflection/collimation apparatus.

i.e., how does one get sufficiently good $\mathrm{R}$ for the efficiency of the reflection/collimation apparatus working optimally, especially if the push per axion goes down because the crucial momentum kick is obtained from speeding up the axion mass-energy packets to light speed, and as their incoming velocities begin to approaches $\mathrm{c}$ the kick gets smaller, reducing the effectiveness of the drive at high velocities?

Lots of engineering headaches abound here, but the even bigger one is an axion that has a mass about $1 / 400,000,000$ of an electron mass, and that there should be about half a trillion of them in each cubic centimeter of space in the vicinity of the earth, more per cc near the galactic center, but only 200,000 per cc in intergalactic space. Figuring that the DM candidates are $10^{5}$ larger in $\mathrm{GeV}$ mass value than electrons, which are in turn about $10^{9}$ times larger than axion candidates, we obtain then almost $10^{14}$ the axion momentum kick if we look at $\mathrm{C}=\mathrm{V}$ contribution. However, as one approached the speed of light, any purported adaptation of Equation (4) would be very, very problematic. We expect at most $1 \mathrm{c}$ values in velocity, which would make the DM convert to Photons for an upgraded Photon rocket not relativistic, but still a huge improvement over simpler versions of the Photon rocket, even say the Eugen Sanger [23] version written above. Furthermore, this is viable and useful to consider, especially when the axion may be now detectable as indicated by C. Rizzo, A-M. Sautivet, et al. (2007) [25].

\section{Further Considerations. Very Important to Keep in Mind. Future Research Directions?}

It is important to keep in mind [9] [10], that further investigations of Dark Matter candidates are imminently feasible. Note that this paper makes use of the "sterile neutrino" paradigm of DM, which may be effectively challenged by extended theories of gravity as emphasized in both [9] [10]. If Sterile neutrinos [26], do not hold, then the work done in [9] [10] must be upheld, and investigated, and this to find candidates for the ram jet as written up in [7], perhaps independently of the Sterile neutrino hypothesis, if [26] is indeed falsified.

\section{Acknowledgements}

This work is supported in part by National Nature Science Foundation of China grant No. 11375279. The author wishes to thank Dr. Eric Davis and Dr. Robert Baker for stimulating his interest in gravitation, and gravitational waves. These interests have lead the author to inquire as to DM properties, and its spill over into possible technology applications, since they are part of the same puzzle, i.e., general space time physics. 


\section{References}

[1] Beckwith, A.W. (2008) The Author Gave Substantially This Presentation in Stockholm, Sweden, in IDM. http://agenda.albanova.se/confRegistrantsDisplay.py/list?confId=355

[2] Hooper, D. (2008) http://agenda.albanova.se/confRegistrantsDisplay.py/list?confId=355

[3] Hooper, D. and Goodenough, L. (2011) Dark Matter Annihilation in the Galactic Center as Seen by the Fermi Gamma Ray Space Telescope. Physics Letters B, 697, 412-428. http://arxiv.org/abs/1010.2752v3 http://dx.doi.org/10.1016/j.physletb.2011.02.029

[4] Meissner, K. and Nicolai, H. (2007) Conformal Symmetry and the Standard Model. Physics Letters B, 648, 312-317. https://arxiv.org/abs/hep-th/0612165 http://dx.doi.org/10.1016/j.physletb.2007.03.023

[5] Drewes, M. (2013) The Phenomenology of Right Handed Neutrinos. International Journal of Modern Physics E, 22, 1330019. https://arxiv.org/abs/1303.6912 http://dx.doi.org/10.1142/s0218301313300191

[6] Lemos, R. (2008) Scientists Say We Will Never Reach the Stars. Wired Magazine (August). http://www.wired.com/science/space/news/2008/08/space_limits

[7] Beckwith, A.W. (2009) https://arxiv.org/vc/arxiv/papers/0810/0810.1493v3.pdf

[8] Beckwith, A.W. (2009) Hypothetical Dark Matter/Axion Rockets: What Can Be Said about Dark Matter in Terms of Space Physics Propulsion. Contributed to SPESIF-2009, Huntsville, 24-27 February 2009. http://arxiv.org/abs/0810.1493 http://dx.doi.org/10.1063/1.3115506

[9] Corda, C. (2009) Interferometric Detection of Gravitational Waves: The Definitive Test for General Relativity. International Journal of Modern Physics D, 18, 2275.

https://arxiv.org/abs/0905.2502 http://dx.doi.org/10.1142/S0218271809015904

[10] Nojiri, S. and Odintsov, S. (2011) Unified Cosmic History in Modified Gravity: From F(R) Theory to Lorentz Non-Invariant Models. Physics Reports, 505, 59. http://arxiv.org/pdf/1011.0544.pdf http://dx.doi.org/10.1016/j.physrep.2011.04.001

[11] Collar, J.I., Miller, D., Rasmussen, J., et al. (2005) CAST, The Solar Axion Search at CERN. http://collargroup.uchicago.edu/projects/axion/index.html

[12] Yang, Q. (2015) Axions and Dark Matter. https://arxiv.org/abs/1509.00673

[13] Kolb, E., Pi, S.-Y. and Raby, S. (1984) Phase Transitions in Supersymmetric Grand Unified Models. In: Fang, L.Z. and Ruffini, R., Eds., Cosmology of the Early Universe, World Press Scientific.

[14] Kolb, E.W. and Turner, M.S. (1990) The Early Universe. Addison-Wesley Publishing Company, The Advanced Book Program, Redwood City.

[15] Muramaya, H. (2006) Physics beyond the Standard Model and Dark Matter. Session LXXXVI of Les Houches.

[16] http://www.ifa.hawaii.edu/ ger/ASTRO-110_sp08/Lecture28_DarkMatter.pdf

[17] Blandford, R.D. and Narayan, R. (1992) Cosmological Applications of Gravitational Lensing. Annual Review of Astronomy and Astrophysics, 30, 311-358.

[18] Roland, S. (2015) A New Model for Sterile Neutrino Dark Matter. https://indico.cern.ch/event/373156/contributions/1793073/attachments/1142692/1637250/ New_Neutrino_DM_Model.pdf 
[19] Madsen, J. (1991) Generalized Tremaine-Gunn Limits for Bosons and Fermions. Physical Review D: Particles and Fields, 44, 999-1006. http://dx.doi.org/10.1103/PhysRevD.44.999

[20] Peebles, P.J.E. (1993) Principles of Physical Cosmology. Princeton Series in Physics.

[21] Adhikari, R., et al., Including Ruchayskiy, O. (2016) A White Paper on keV Sterile Neutrino Dark Matter. http://arxiv.org/abs/1602.04816

[22] Taoso, M., Bertone, G. and Masiero, A. (2008) Dark Matter Candidates: A Ten-Point Test. JCAP 0803:022,2008. https://arxiv.org/abs/0711.4996

[23] Gulevich, A., Ivanov, E., Kukharchuk, O., Poupko, V.Y. and Zrodnikov, A.V. (2001) Applications of Nuclear Photon Engines for Deep Space Exploration. AIP Conference Proceedings, 552, 957-962. http://dx.doi.org/10.1063/1.1358034

[24] Sikivie, P. (1983) Experimental Tests of the "Invisible" Axion. Physical Review Letters, 51, 1415. http://dx.doi.org/10.1103/PhysRevLett.51.1415

[25] Rizzo, C., Sautivet, A.-M., et al. (2007) No Light Shining through a Wall. CRNS: France, arXiv. http://arxiv.org/pdf/0707.1296

[26] Ruchayskiy, O. (2007) Restrictions on Sterile Neutrino Parameters from Astrophysical Observations. ArXiv 0704.3215v1.

Submit or recommend next manuscript to SCIRP and we will provide best service for you:

Accepting pre-submission inquiries through Email, Facebook, LinkedIn, Twitter, etc.

A wide selection of journals (inclusive of 9 subjects, more than 200 journals)

Providing 24-hour high-quality service

User-friendly online submission system

Fair and swift peer-review system

Efficient typesetting and proofreading procedure

Display of the result of downloads and visits, as well as the number of cited articles

Maximum dissemination of your research work

Submit your manuscript at: http://papersubmission.scirp.org/ 